\title{
Thai Language Conventions
}

THIs BOOK adheres to the phonetic transcription for most Thai words, but without tonal marks. This practice follows the "General System of Phonetic Transcription of Thai Characters into Roman" devised by the Royal Institute, Bangkok, in 1954. Moreover, because of the constraints of typesetting, the superscript and subscript marks of certain vowels and consonants are not shown.

Exceptions are those names which have been transcribed by various other systems or perhaps no system at all. In the case of a name which is widely known or which can be checked, the owner's transcription has been adhered to. Otherwise, the spelling follows the system of romanization mentioned above. The English names of certain Thai kings, princes, and nobles, as they are known among historians (Mongkut, Chulalongkorn, Vajiravudh) have been adopted rather than the official lengthy titles. Likewise I refer to Damrong, Phichit, Prachak, Wachirayan, Thiphakorawong, and so on rather than their full titles and names. In most cases, however, their ranks such as Prince, Phraya, Chao Phraya, and their longer titles are given in the first reference to each.

Finally, as in conventional usage, Thai people are referred to by their first names while Westerners are referred to by their surnames. In the bibliography, Thai names are entered according to first names. 
